\title{
An MRS- and PET-guided biopsy tool for intraoperative neuronavigational systems
}

\author{
Matthew Grech-Sollars, PhD, ${ }^{1,2}$ Babar Vaqas, BSc, BMBCh(Oxon), FRCS(SN), ${ }^{3}$ \\ Gerard Thompson, FRCR, PhD, ${ }^{4}$ Tara Barwick, MBChB, MSc, MRCP, FRCR, ${ }^{1,5}$ \\ Lesley Honeyfield, DCR(R), ${ }^{1}$ Kevin O'Neill, MBBS, FRCS(SN), ${ }^{3}$ and \\ Adam D. Waldman, MBBChir(Cantab), FRCP, FRCR, PhD'1,2
}

\begin{abstract}
Departments of ${ }^{1}$ Imaging and ${ }^{3}$ Neurosurgery, Imperial College Healthcare NHS Trust; ${ }^{5}$ Department of Surgery and Cancer, and 2Division of Brain Sciences, Imperial College London; and ${ }^{4}$ Department of Neuroradiology, Salford Royal NHS Foundation Trust, Salford, United Kingdom
\end{abstract}

\begin{abstract}
OBJECTIVE Glioma heterogeneity and the limitations of conventional structural MRI for identifying aggressive tumor components can limit the reliability of stereotactic biopsy and, hence, tumor characterization, which is a hurdle for developing and selecting effective treatment strategies. In vivo MR spectroscopy (MRS) and PET enable noninvasive imaging of cellular metabolism relevant to proliferation and can detect regions of more highly active tumor. Here, the authors integrated presurgical PET and MRS with intraoperative neuronavigation to guide surgical biopsy and tumor sampling of brain gliomas with the aim of improving intraoperative tumor-tissue characterization and imaging biomarker validation.
\end{abstract}

METHODS A novel intraoperative neuronavigation tool was developed as part of a study that aimed to sample highcholine tumor components identified by multivoxel MRS and ${ }^{18} \mathrm{~F}$-methylcholine PET-CT. Spatially coregistered PET and MRS data were integrated into structural data sets and loaded onto an intraoperative neuronavigation system. High and low choline uptake/metabolite regions were represented as color-coded hollow spheres for targeted stereotactic biopsy and tumor sampling.

RESULTS The neurosurgeons found the 3D spherical targets readily identifiable on the interactive neuronavigation system. In one case, areas of high mitotic activity were identified on the basis of high ${ }^{18} \mathrm{~F}$-methylcholine uptake and elevated choline ratios found with MRS in an otherwise low-grade tumor, which revealed the possible use of this technique for tumor characterization.

CONCLUSIONS These PET and MRI data can be combined and represented usefully for the surgeon in neuronavigation systems. This method enables neurosurgeons to sample tumor regions based on physiological and molecular imaging markers. The technique was applied for characterizing choline metabolism using MRS and ${ }^{18} \mathrm{~F} \mathrm{PET;} \mathrm{however,} \mathrm{this}$ approach provides proof of principle for using different radionuclide tracers and other MRI methods, such as MR perfusion and diffusion.

https://thejns.org/doi/abs/10.3171/2016.7.JNS16106

KEY WORDS intraoperative imaging; MRS; PET; surgical planning; glioma; surgical technique

$\mathrm{H}$ IGH-RESOLUTION MR images, acquired presurgically, are commonly used in intraoperative neuronavigation systems to guide stereotactic biopsy and tumor resection..$^{2}$ Regions of pathological contrast enhancement on Gd-enhanced T1-weighted images and T2-dependent signal abnormality on T2-weighted and FLAIR images are typically used to define surgical targets. Spatial heterogeneity in gliomas and poor biological specificity of these conventional MRI sequences limit tumor characterization and, hence, clinical decision making for postsurgical phases of treatment. Indeed, the histopathological diagnosis from ste- reotactic biopsy using standard imaging has been shown to disagree with that from the resected tumor in almost $50 \%$ of patients when reviewed by a single histopathologist. ${ }^{6}$

Imaging modalities that can detect the most aggressive tumor elements and delineate tumor margins are being developed. Multimodal MRI, which incorporates quantitative and physiological methods such as in vivo MR spectroscopy (MRS), and molecular imaging with PET tracers yield additional information on tumor metabolism and cellular turnover and have been shown to augment the information available from structural imaging. Validation 
of these imaging biomarkers against tumor biology is essential, and integrating such images in neurosurgical planning is required.

The use of a number of different imaging techniques was explored previously for guiding neurooncological surgery. ${ }^{10,18}$ MRS data also have been incorporated into neuronavigational systems; $;^{10,17,20}$ however, those studies did not involve direct spatial location of MRS information directly onto high-resolution T1-weighted images. MRS also requires offline processing, analysis, and interpretation to exclude artifacts and errors ${ }^{7}$ before it can be used to identify surgical targets. PET has also been applied to guiding biopsy and surgical tumor resection. ${ }^{8,11,15}$

Here, we describe the successful integration of MRS and PET into intraoperative neuronavigational systems for advanced biopsy and tumor-sample targeting in a clinical environment.

\section{Methods}

A surgical planning tool was required as part of a prospective single-center cohort study that aimed to compare ${ }^{18}$ F-methylcholine (FMC) uptake on PET and elevated choline-containing compound (Cho) levels on MRS against tissue markers of Cho metabolism. (Throughout the article, Cho refers to choline-containing compounds as defined in MRS, and FMC refers to ${ }^{18} \mathrm{~F}$-methylcholine in PET; choline is a generic term that refers to either MRS or PET.) The technique presented here was developed on the cohort of 14 patients recruited for that study. These patients had a diagnosis of primary brain tumor on imaging and were undergoing a biopsy or resection of their tumor within a few months of imaging. Surgery took place between 1 day and 8 weeks after the MRS and PET images were acquired. Ethical approval was given by the National Research Ethics Service Committee London-Fulham, and informed consent was obtained from each participant. All data were anonymized in accordance with the UK Data Protection Act.

\section{Image Acquisition}

MR images were acquired on a 3-T Verio VB19 MRI system (Siemens) using a 32-channel head coil. The MRI protocol included pre-Gd and post-Gd high-resolution 3D volumetric T1-weighted imaging (voxel size $1 \times 1 \times 1 \mathrm{~mm}$ ) and multivoxel MRS using 2D chemical-shift imaging (CSI) point-resolved spectroscopy (PRESS) (TE $30 \mathrm{msec}$, TR $1700 \mathrm{msec}$, voxel size $10 \times 10 \times 15 \mathrm{~mm}$ ); the total acquisition time for one CSI slice was 7 minutes. PET-CT was performed on a Siemens Biograph 6 scanner with CT at $120 \mathrm{kV}, 5 \mathrm{~mA}$, followed by a bolus injection of $285 \mathrm{MBq}$ of FMC (PETNET Solutions) using a dedicated 45-minute brain dynamic list-mode acquisition.

\section{Image Analysis and Integration and Surgical Planning}

MRS data were analyzed using proprietary Siemens software and TARQUIN, which uses time domain-based algorithms for spectroscopic metabolite analysis..$^{21}$ Ratios of Cho-containing compounds to creatine and phosphocreatine $(\mathrm{Cr})$ were calculated for each MRS voxel.

Time-activity curves from dynamically acquired FMC
PET allowed time-averaged images to be calculated from averaged signal (last 10 minutes of dynamic acquisition from tracer injection) during a pseudo-steady-state phase (Hermes Diagnostics). Regions of tumoral uptake were identified visually from appropriately windowed images.

An imaging scientist with experience in physiological imaging of gliomas processed and analyzed the MRS and PET data after discussion with neuroradiologists experienced in MRS and PET-CT imaging of gliomas. Voxels that indicated high and low $\mathrm{Cho/Cr}$ ratios on MRS and high uptake on PET were identified.

In the initial cases, the locations at which MRS and PET were to be sampled were discussed with the surgeons, and a hard copy of images showing the area to sample was provided. However, this process was found to be inaccurate and unreliable; hence, a surgical planning tool that could define regions to sample intraoperatively was required.

Spatial information was extracted from the DICOM MRS data set so that the targets could be overlaid on one of the images acquired in the same imaging session. The targets were overlaid on high-resolution postcontrast T1weighted structural images, which were acquired immediately before the MRS sequence to minimize the effects of registration error caused by any patient motion.

The first attempt at presenting these regions to the surgeons involved using a cross-type marker on the preoperative axial images on one image slice. However, when they were viewed at an oblique angle, these markers were not clearly visible, and an improved method for representing the biopsy targets was required.

To generate readily visible surgical targets, image masks of hollow spheres were constructed around the individual MRS voxels already identified using bespoke software written in MATLAB (Fig. 1).

Similarly, hollow-sphere image masks were created for areas that showed high and low choline uptake on PET imaging by first registering the PET images to the MR images using Vinci software (http://www.nf.mpg.de/ vinci3/) and then identifying the locations of high FMC PET uptake. All of the regions selected for biopsy were then overlaid onto the presurgical high-resolution T1weighted image using a color-coded system to indicate which region identified with MRS or PET corresponded to each sphere. Thus, multiple target tumor-sampling areas were presented to the surgeons using color coding to identify if a sample was taken from an area identified through the PET data, the MRS data, or both. Masks were overlaid using a custom-written MATLAB script, and images were saved in DICOM format. The resultant image (Fig. 1 upper right) was then loaded to the neuronavigational system.

\section{Intraoperative Guidance}

The target images were loaded onto neuronavigational systems such that the color-coded spheres were easily visible in all planes and at oblique angles (Fig. 2). One of 2 neuronavigational systems was used for 13 patients who underwent surgery in this study: a 3D ultrasound neuronavigation system (SonoWand Invite) ${ }^{5}$ in 9 patients (Fig. 2) or a Medtronic StealthStation in 4 patients (Fig. 3). For the StealthStation and an older SonoWand system, grayscale spheres were used to indicate the different areas in these 


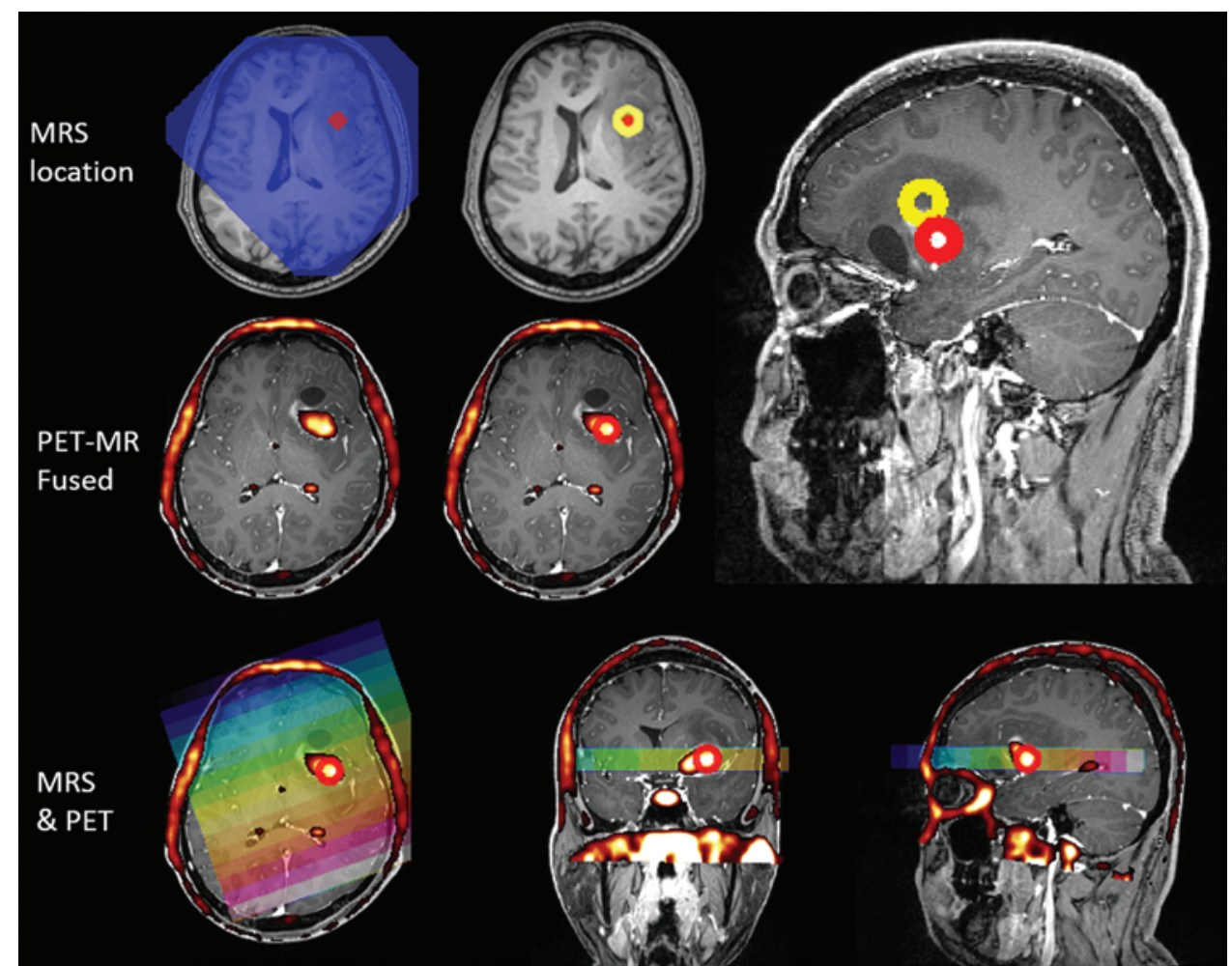

FIG. 1. Integration of PET and MRS into a surgical plan. MRS data are analyzed, and the voxel to be sampled is selected (red). A hollow sphere is then placed around this voxel (yellow) to indicate the target area within the center of the sphere to biopsy. PET data are analyzed also, and the area to be sampled is indicated using a hollow sphere (red), which is also added to the surgeryplanning image (upper right). By use of this system, MRS and PET data can also be combined to show the spatial correlation between the 2 modalities. Images are from a 34-year-old male patient with a histological diagnosis of WHO Grade IV glioblastoma. Figure is available in color online only.

cases because these systems did not accept color DICOM images.

In addition to generating the image masks consisting of hollow spheres for each voxel, the software also generated a slab of all the voxel locations that could be overlaid on top of the structural MR and PET images to retrospectively identify and confirm which voxel of MRS data corresponded to each of the samples taken during surgery.

After the registration of this integrated data set with the patient's cranial position at the time of surgery, the surgical incision and craniotomy were planned to enable optimal access to the tumor-sampling areas identified. In each case, 1-5 stereotactic biopsies targeted to regions with high and low $\mathrm{Cho} / \mathrm{Cr}$ ratios and FMC uptake were performed before any resection to minimize the effects of brain shift on the accuracy of the navigation system during surgery. A neuronavigation-compatible passive biopsy needle was used to obtain frameless stereotactic tissue samples.

When a tumor sample was taken from the center of a sphere, the location was recorded on the navigation system together with the sample number to correlate accurately with histology. In cases in which the sample was not taken from the center of the sphere, the recorded positional data enabled the exact location of the sample to be determined. The corresponding MRS voxel and PET results were identified by overlaying the PET images and MRS voxel locations, as shown in Fig. 1.

\section{Results}

After a number of iterations to develop the method, the coregistration and integration of presurgical MRS and PET data into the neuronavigation systems was successful.

The spherical targets were readily identifiable on the neuronavigational system in the operating theater environment, which enabled confident targeting of biopsy and tumor sampling. This method was developed after initial trials of using cross-type targets to represent integrated imaging data failed to suitably present the tumor-sampling targets in the operating theater. In that case, the neurosurgeons found it difficult to identify the targets (which were $5-10 \mathrm{~mm}$ across but effectively only $1 \mathrm{~mm}$ thick) and plan the biopsy approach, because the targets readily disappeared out of the visualized imaging plane, particularly when they were viewed at an oblique angle rather than on an axial plane. In contrast, the spherical targets provided simple visualizations that were identified easily at any angle and on any of the image planes. Color coding these spheres provided a method for indicating to the neurosurgeons what each of the spheres described, and the hollow part of the sphere represented the epicenter of the MRS voxel $(10 \times 10 \times 15 \mathrm{~mm})$.

A screenshot of the 3D neuronavigational ultrasound system taken during biopsy performed using these images is shown in Fig. 2. In that patient, the red hollow sphere indicated areas of high Cho, and the blue hollow sphere 


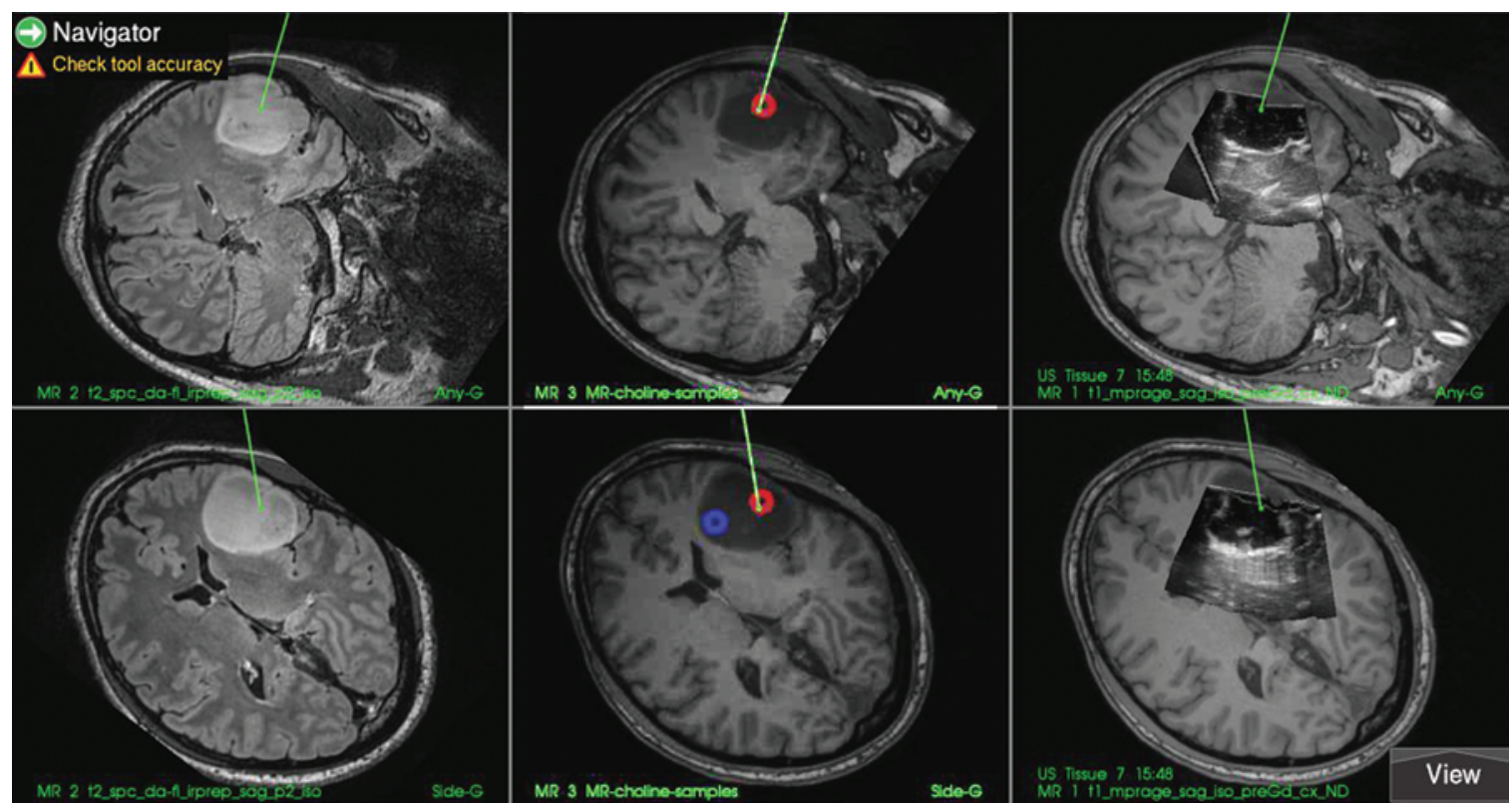

FIG. 2. System in use in the operating theater. A screenshot from the SonoWand system shows the presurgery MR images (left), the surgery plan (center), and the ultrasound overlaid image (right) at 2 different oblique angles (upper and lower). The ultrasound images provide near real-time surgery guidance. The 3D color-coded hollow spheres enable easy identification of targets at any angle. The green markers indicate from where the sample was taken during surgery. Images are from a 34-year-old male patient with a histological diagnosis of WHO Grade II astrocytoma. Figure is available in color online only.

indicated areas of low Cho. Planning images are shown together with the preoperative images (T2 weighted in the case of the patient shown in the screenshot).

In one case, a region of high Cho metabolism within a nonenhancing lesion corresponded to a region of early focal malignant transformation on a background of a WHO Grade II astrocytoma within low Cho components. The sample in this area showed mitotic activity indicative of a transforming tumor (WHO Grade II-III).

\section{Discussion}

Gliomas are frequently spatially heterogeneous, and the ability to identify and accurately sample the most aggressive tumor components is essential for correct tissue classification and postsurgical treatment stratification, which are particularly important when the tumor location permits only limited-sampling partial resection or stereotactic biopsy and with nonenhancing, atypical, and multicentric lesions. There is a significant body of evidence to indicate that physiological MRI methods and molecular imaging with PET enable the detection of tumor phenotype and heterogeneity, which are not apparent with routine structural MRI. ${ }^{14}$

\section{Previously Used Techniques}

The use of "advanced" imaging to guide biopsy, however, is less well established. In particular, previous studies were not effective in prospectively correlating histopathology and MRS accurately. A number of different approaches have been described in published reports.

Some studies recorded the biopsy site on T1- or T2weighted images during surgery and retrospectively correlated them with MRS data. ${ }^{3,4}$ This technique is not usable in a prospective cohort in which the aim is to take samples from specific areas identified with advanced imaging.

Other reports described hard-copy MR images presented to the surgeon in the operating theater who then used the hard copy to identify the MRS voxel location. ${ }^{9}$ This method is prone to increased errors in target sampling, and satisfactory postsurgical verification of the biopsy location is also not possible. As part of developing the technique presented here, we experimented with this method, which proved cumbersome in practice and prolonged the surgical procedure unacceptably. Identifying targets was particularly difficult when the optimum plane of imaging for the surgical approach differed from the axial plane on which spectroscopy was acquired. The required sampling locations in these cases could not be represented on the correct plane on the hard-copy images.

Intraoperative MRI has been used to target tissue based on single-voxel MRS data acquired with structural MRI. ${ }^{13}$ This method has the potential advantage of enabling correction for brain shift by updating the metabolically defined target during surgery. However, intraoperative MRI is costly and available in very few centers, often requires the presence of a radiologist to analyze the images, and is recognized to lengthen the duration of surgical procedures. Intraoperative MRS is also technically challenging and prone to artifacts from tissue-air interfaces around the exposed tumor ${ }^{1}$ that can markedly degrade the metabolic data acquired.

A fourth method that has been described involves acquiring a separate 5-slice T1-weighted image for the location of MRS and copying the MRS results onto this 5-slice T1-weighted image before loading the MRS data into a color map of the $\mathrm{Cho} / \mathrm{N}$-acetylaspartate ratio onto the neuronavigational system. ${ }^{17,20}$ This method is less 


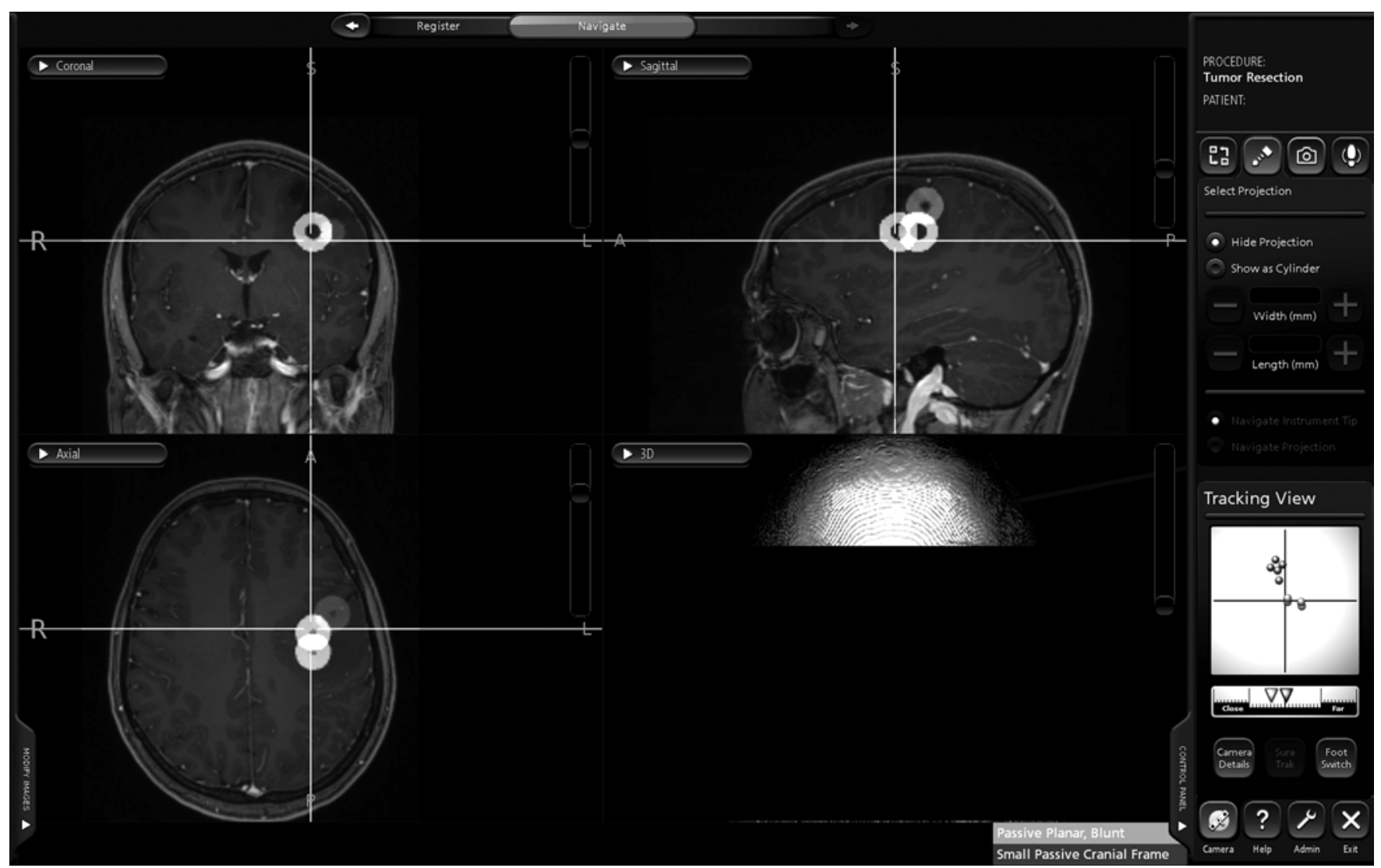

FIG. 3. System in use in the operating theater. A screenshot from the StealthStation system shows the surgery plan on the presurgery MR images. In this case, grayscale images were required. The crosshairs mark the area from which the biopsy was taken. Images are from a 23-year-old female patient with a histological diagnosis of WHO Grade II oligodendroglioma.

accurate than using the spatial localization information available within the MRS data set, and it increases the complexity of the surgery by introducing an imageanalysis step at the point of performing the biopsy. It also requires pre-analysis of the MRS data to ensure that the quality of the data within the various voxels from which the color maps are derived are sufficient for meaningful interpretation.

In some regards, representing PET on neuronavigational systems is more straightforward than representing MRS, because it generates a series of images rather than spectra. That said, the appropriate selection of intensity level and width "windows" is necessary to enable visualization of different regions of abnormal tracer uptake. Even when the intensity level and width window had been optimized on a PET workstation, however, we found that regions of elevated uptake were not always conspicuous when PET images were fused directly onto the neuronavigational system. The close inspection and interpretation of the images that were required prolonged the surgical procedure. Pre-selection and representation of targets using the hollow-sphere method circumvented this problem.

\section{The Developed Tool}

We developed and implemented a usable method for MRS- and PET-guided biopsy that involves the following 3 steps.

\section{Offline Processing, Analysis, and Interpretation of Data}

Multivoxel MRS (Fig. 4) enables metabolite profiles to be mapped over a sizeable and defined volume of tissue. $\mathrm{Cho} / \mathrm{Cr}$ and $\mathrm{Cho} / \mathrm{N}$-acetylaspartate ratios are known to be different among low- and high-grade tumors, ${ }^{19}$ and highCho regions provide potential indicators of aggressive tumor components in diffuse gliomas. The spectra require offline processing and analysis and an understanding of technical factors and artifacts that are critical for reliable interpretation. Therefore, processing and analysis were performed by a neuroradiologist and imaging scientist experienced with in vivo MRS, using proprietary and opensource software for processing, analysis, and manual voxel target selection.

High- and low-uptake targets also were selected manually from appropriately windowed static FMC PET images derived from dynamic acquisition.

\section{Reliable Coregistration of Physiological/Molecular Images With High-Resolution Structural Data Sets}

Spectroscopy generates biochemical maps that lack structural features, which precludes the use of rigid-body registration methods that are typically used to fuse functional and structural images. We therefore developed software to extract coordinates from the spectroscopy-acquisition parameters, which could be coregistered with the volumetric MRI data sets. 


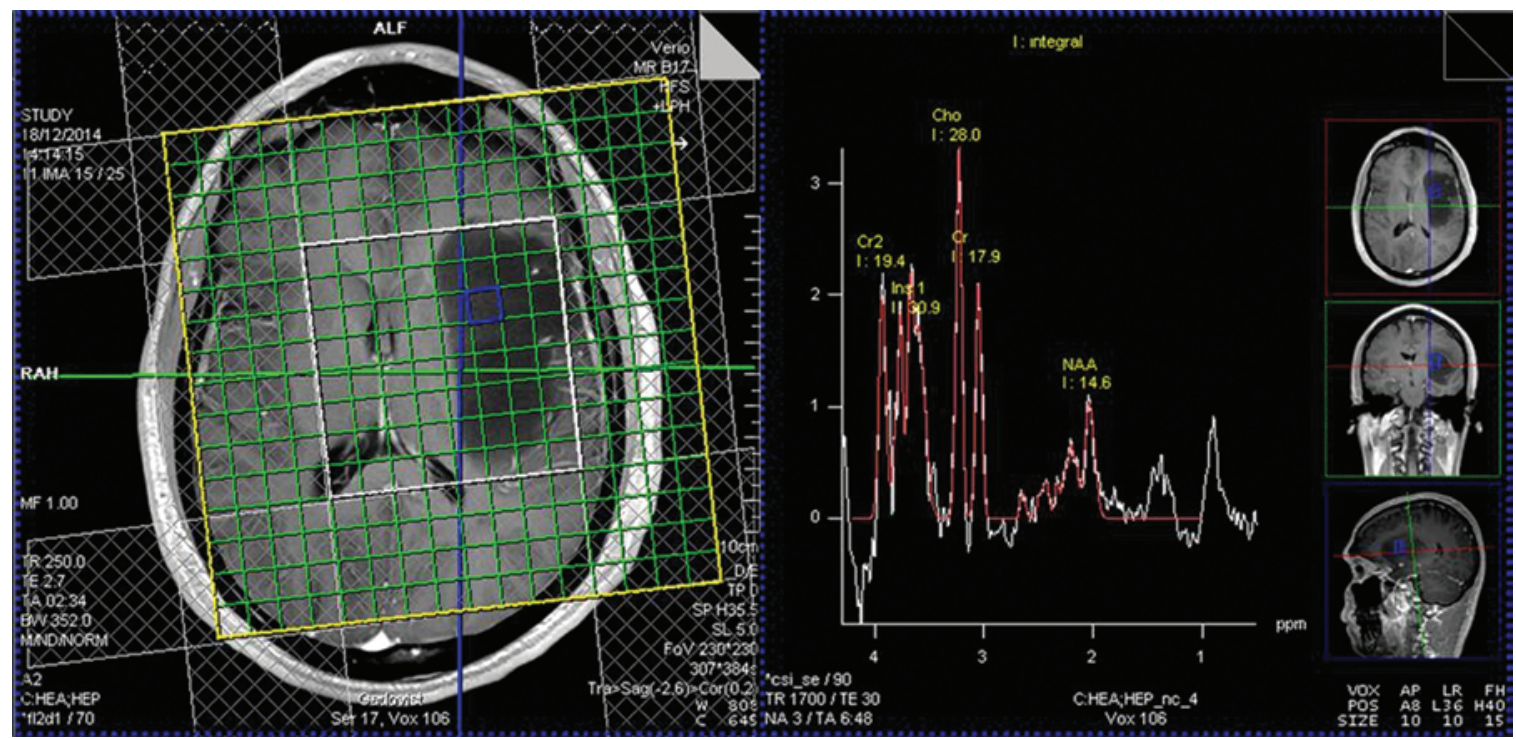

FIG. 4. MRS data. Multivoxel MRS data are acquired over large areas of tumor, and a spectrum showing the different metabolites can be seen in each voxel area. In this case, areas of moderate Cho were observed in the tumor. Figure is available in color online only.

Coregistration of PET and structural high-resolution MRI data is a more straightforward process, and it was carried out using well-established rigid-body algorithms available in Vinci.

\section{Generation of Targets That Can Be Integrated and Used in Neuronavigation Systems in the Operating Theater}

Our approach was to synthesize hollow virtual-target spheres centered on the feature of interest in MRS or PET images. Color coding enabled tissue samples to be labeled simply and subsequently correlated with imaging features.

One of our priorities was to develop a methodology that optimizes tissue sampling with minimal negative impact on the complexity and length of surgical procedures. Such a guidance tool can be used mainly in the following 2 ways.

The first use is multimodal imaging to guide biopsy and tumor sampling to optimize glioma tissue characterization for clinical stratification. Although this was not a primary aim of our study, in one patient, high-Cho regions revealed histological evidence of malignant transformation in a WHO Grade II lesion that altered subsequent clinical management. This approach can also be used to optimize the resection of aggressive tumor components and evaluate its effects on survival.

The second use is for validating current and emerging imaging biomarkers against tissue biomarkers in defined regions of tissue. This validation was the primary aim of the research study to which patients were recruited and for which this tool was initially developed.

\section{Limitations}

Considerable processing and analyses are required for presurgical planning, which is labor intensive and requires both specialist neuroradiology and imaging clinical scientist expertise. This requirement is particularly critical for patients with a short lead time in between imaging and surgery. Furthermore, the formats of imaging data produced by different MRI platforms and required by commercial neuronavigational systems might be vendor specific, and processes might need modification for individual combinations of equipment. For example, our StealthStation and an older SonoWand system would not allow the display of color-coded images. Moreover, we developed the tool for use on Siemens MRI data sets, and it is likely that modifications to the MATLAB code would be required for data sets from other vendors. The code we developed to extract the MRS spatial locations can be provided on request for other researchers to test.

The exact accuracy and precision with which regions of interest, chosen as targets, are represented in the neuronavigation system are difficult to determine reliably. Our targets are coregistered on presurgical images and do not take into account $\geq 1-\mathrm{cm}$ brain shifts that can occur during an operation. ${ }^{2,16}$ Because the stereotactic biopsies were performed before tumor resection, the degree of target misalignment is likely to have been small, although a minor shift might have occurred as a result of the craniotomy alone. Furthermore, the exact location at which the biopsy was performed cannot be verified after the tumor is resected because it cannot be imaged after resection. Thus, the accuracy of the tool depends on that of the neuronavigational system with which it is used, and factors that limit accuracy of the system need to be considered. ${ }^{12}$

\section{Conclusions}

We have developed a targeted PET- and MRS-guided stereotactic biopsy tool that uses intraoperative neuronavigation that involves a combination of proprietary and bespoke software to analyze and integrate physiological imaging data and novel presentation of biopsy targets as hollow spheres that can readily be identified in the operating environment. Our preliminary experiences indicate that this method can be used to improve the diagnostic accuracy of stereotactic biopsy, provided that the advanced 
imaging techniques (in this case, PET and MRS) included in the planning system can indicate more aggressive areas of an otherwise heterogeneous tumor. Our results also provide proof of principle for spatially correlated validation of a variety of imaging biomarkers against tissue features.

\section{Acknowledgments}

We thank Ms. Sophie Camp for her help in trialling the images on the Medtronic StealthStation. We also thank Dr. Katherine Ordidge and Mr. Daniel Darian for their help in this study. MRI and PET imaging were performed at the Imperial College Clinical Imaging Facility.

This work was funded in part by the National Institute for Health Research Imperial Comprehensive Biomedical Research Centre. Dr. Grech-Sollars is funded partly by The Brain Tumour Charity and Brain Tumour Research Campaign.

\section{References}

1. Abernethy LJ, Avula S, Hughes GM, Wright EJ, Mallucci CL: Intra-operative 3-T MRI for paediatric brain tumours: challenges and perspectives. Pediatr Radiol 42:147-157, 2012

2. Black PM, Alexander E III, Martin C, Moriarty T, Nabavi A, Wong TZ, et al: Craniotomy for tumor treatment in an intraoperative magnetic resonance imaging unit. Neurosurgery 45:423-433, 1999

3. Croteau D, Scarpace L, Hearshen D, Gutierrez J, Fisher JL, Rock JP, et al: Correlation between magnetic resonance spectroscopy imaging and image-guided biopsies: semiquantitative and qualitative histopathological analyses of patients with untreated glioma. Neurosurgery 49:823-829, 2001

4. Dowling C, Bollen AW, Noworolski SM, McDermott MW, Barbaro NM, Day MR, et al: Preoperative proton MR spectroscopic imaging of brain tumors: correlation with histopathologic analysis of resection specimens. AJNR Am J Neuroradiol 22:604-612, 2001

5. Gronningsaeter A, Kleven A, Ommedal S, Aarseth TE, Lie T, Lindseth F, et al: SonoWand, an ultrasound-based neuronavigation system. Neurosurgery 47:1373-1380, 2000

6. Jackson RJ, Fuller GN, Abi-Said D, Lang FF, Gokaslan ZL, Shi WM, et al: Limitations of stereotactic biopsy in the initial management of gliomas. Neuro Oncol 3:193-200, 2001

7. Kreis R: Issues of spectral quality in clinical 1H-magnetic resonance spectroscopy and a gallery of artifacts. NMR Biomed 17:361-381, 2004

8. Levivier M, Goldman S, Pirotte B, Brucher JM, Balériaux D, Luxen A, et al: Diagnostic yield of stereotactic brain biopsy guided by positron emission tomography with $\left[{ }^{18} \mathrm{~F}\right]$ fluorodeoxyglucose. J Neurosurg 82:445-452, 1995

9. McKnight TR, von dem Bussche MH, Vigneron DB, Lu Y, Berger MS, McDermott MW, et al: Histopathological validation of a three-dimensional magnetic resonance spectroscopy index as a predictor of tumor presence. J Neurosurg 97:794802,2002

10. Mert A, Kiesel B, Wöhrer A, Martínez-Moreno M, Minchev G, Furtner J, et al: Introduction of a standardized multimodality image protocol for navigation-guided surgery of suspected low-grade gliomas. Neurosurg Focus 38(1):E4, 2015

11. Misch M, Guggemos A, Driever PH, Koch A, Grosse F, Steffen IG, et al: ${ }^{18}$ F-FET-PET guided surgical biopsy and resection in children and adolescence with brain tumors. Childs Nerv Syst 31:261-267, 2015

12. Orringer DA, Golby A, Jolesz F: Neuronavigation in the surgical management of brain tumors: current and future trends. Expert Rev Med Devices 9:491-500, 2012

13. Pamir MN, Özduman K, Yıldız E, Sav A, Dinçer A: Intraoperative magnetic resonance spectroscopy for identification of residual tumor during low-grade glioma surgery: clinical article. J Neurosurg 118:1191-1198, 2013
14. Peet AC, Arvanitis TN, Leach MO, Waldman AD: Functional imaging in adult and paediatric brain tumours. Nat Rev Clin Oncol 9:700-711, 2012

15. Pirotte B, Goldman S, Dewitte O, Massager N, Wikler D, Lefranc F, et al: Integrated positron emission tomography and magnetic resonance imaging-guided resection of brain tumors: a report of 103 consecutive procedures. J Neurosurg 104:238-253, 2006

16. Roberts DW, Hartov A, Kennedy FE, Miga MI, Paulsen KD: Intraoperative brain shift and deformation: a quantitative analysis of cortical displacement in 28 cases. Neurosurgery 43:749-760, 1998

17. Stadlbauer A, Moser E, Gruber S, Nimsky C, Fahlbusch R, Ganslandt O: Integration of biochemical images of a tumor into frameless stereotaxy achieved using a magnetic resonance imaging/magnetic resonance spectroscopy hybrid data set. J Neurosurg 101:287-294, 2004

18. Tanaka Y, Nariai T, Momose T, Aoyagi M, Maehara T, Tomori T, et al: Glioma surgery using a multimodal navigation system with integrated metabolic images. J Neurosurg 110:163-172, 2009

19. Tong T, Yang Z, Chen JW, Zhu J, Yao Z: Dynamic ${ }^{1} H-M R S$ assessment of brain tumors: a novel approach for differential diagnosis of glioma. Oncotarget 6:32257-32265, 2015

20. Widhalm G, Krssak M, Minchev G, Wöhrer A, TraubWeidinger T, Czech T, et al: Value of ${ }^{1} \mathrm{H}$-magnetic resonance spectroscopy chemical shift imaging for detection of anaplastic foci in diffusely infiltrating gliomas with non-significant contrast-enhancement. J Neurol Neurosurg Psychiatry 82:512-520, 2011

21. Wilson M, Reynolds G, Kauppinen RA, Arvanitis TN, Peet AC: A constrained least-squares approach to the automated quantitation of in vivo ${ }^{1} \mathrm{H}$ magnetic resonance spectroscopy data. Magn Reson Med 65:1-12, 2011

\section{Disclosures}

The authors report no conflict of interest concerning the materials or methods used in this study or the findings specified in this paper.

\section{Author Contributions}

Conception and design: Grech-Sollars, Thompson, Honeyfield. Acquisition of data: Grech-Sollars, Honeyfield, Waldman. Analysis and interpretation of data: Grech-Sollars, Vaqas, Thompson, Waldman. Drafting the article: Grech-Sollars, Waldman. Critically revising the article: Vaqas, Barwick, Waldman. Reviewed submitted version of manuscript: Vaqas. Approved the final version of the manuscript on behalf of all authors: Grech-Sollars. Administrative/technical/material support: Grech-Sollars, Thompson, Honeyfield. Study supervision: O'Neill, Waldman.

\section{Supplemental Information \\ Previous Presentations}

Portions of this work were presented as an oral presentation at the British Society of Neuroradiologists Annual Conference, Sheffield, United Kingdom, October 9, 2015; in poster form at the annual meeting of the ISMRM British Chapter, London, United Kingdom, September 10, 2015; and also in poster form at the 20th Annual Scientific Meeting of the Society for Neuro-Oncology, San Antonio, Texas, November 21, 2015.

\section{Current Affiliation}

Prof. Waldman: Centre for Clinical Brain Sciences, University of Edinburgh, United Kingdom.

\section{Correspondence}

Matthew Grech-Sollars, Radiological Sciences Unit, 2nd Fl. North, Charing Cross Hospital, Fulham Palace Rd., London W6 8RF, United Kingdom. email: m.grech-sollars@imperial.ac.uk. 